Revista Interdisciplinaria de Humanidades, Educación, Ciencia y Tecnología

Año VII. Vol. VII. N². Edición Especial II. 2021

Hecho el depósito de ley: pp201602FA4721

ISSN-L: 2542-3029; ISSN: 2610-802X

Universidad Nacional Experimental Francisco de Miranda (UNEFM). Santa Ana de Coro. Venezuela

Percy Ramírez-Medrano

\title{
Sistema de gestión de calidad ISO 9001:2015 para optimizar los procesos críticos en instituciones universitarias
}

\section{ISO 9001: 2015 Quality Management System to optimize critical processes in university institutions}

\author{
Percy Ramírez-Medrano \\ pe.ramirez.med@gmail.com \\ Universidad Nacional Federico Villarreal, Lima \\ Perú \\ https://orcid.org/0000-0002-4994-8770
}

Recibido: 10 de agosto de 2021

Aprobado: 01 de septiembre de 2021 


\title{
RESUMEN
}

La investigación tiene por objetivo determinar el impacto que ejerce la implementación de un Sistema de Gestión de la Calidad ISO 9001:2015 en el proceso de licenciamiento institucional de la Universidad Nacional Daniel Alcides Carrión. De tipo explicativa. Se destaca que el tratamiento aplicado ejerció modificación sobre la gestión de calidad, llevada a cabo por la población estudiad, por lo tanto, se acepta H1 y se rechaza H0. Con un enfoque basado en procesos con información relevante y medible, y a la vez permite retroalimentar los procesos de gestión de la calidad para determinar los procesos críticos y gestionar los riesgos y aplicar las propuestas de solución, para así lograr la mejora continua en la gestión de la calidad en beneficios de los estudiantes, docentes, personal administrativo, grupos de interés, partes interesadas y demás relacionados con el que hacer universitario además le permite un posicionamiento en la sociedad.

Descriptores: Calidad de la educación; pertinencia de la educación; evaluación de la educación. (Palabras tomadas del Tesauro UNESCO).

\begin{abstract}
The research aims to determine the impact of the implementation of an ISO 9001: 2015 Quality Management System in the institutional licensing process of the Daniel Alcides Carrión National University. Explanatory type. It is highlighted that the applied treatment exerted modification on the quality management, carried out by the studied population, therefore, $\mathrm{H} 1$ is accepted and $\mathrm{H} 0$ is rejected. With a process-based approach with relevant and measurable information, and at the same time it allows feedback on quality management processes to determine critical processes and manage risks and apply solution proposals, in order to achieve continuous improvement in the management of the quality of benefits for students, teachers, administrative staff, interest groups, interested parties and others related to what to do in college also allows a position in society.
\end{abstract}

Descriptors: Educational quality; educational relevance; educational evaluation. (Words taken from the UNESCO Thesaurus). 


\section{INTRODUCCIÓN}

Generalmente los sistemas de Gestión de la Calidad implementados toman en cuenta los requisitos de la norma ISO 9001, lo que pretendemos es juntar los requisitos de la norma ISO 9001:2015 con los requerimientos de las condiciones básicas de calidad del modelo de licenciamiento institucional planteado por la Superintendencia Nacional de Educación SUNEDU y la estructura funcional de la Universidad Nacional Daniel Alcides Carrión, el cual ayudará al aseguramiento de la calidad según la D.S. 0016-2015-MINEDU.

En el Perú, con la promulgación de la ley universitaria 30220, en julio del 2014, se crea un marco para el aseguramiento de la calidad universitaria basada en cuatro principios, la Acreditación de programas de estudio e instituciones, el licenciamiento institucional y se entiende que luego empieza el licenciamiento de programas de estudio, el sistema de información que pretende contar con un sistema de interacción de datos de entidades del estado y el sistema universitario, el desempeño que busca la competencia de todos los involucrados en la formación de estudiantes de nivel superior universitario y no universitario.

En promedio desde el 2005 la Asamblea Nacional de Rectores ANR, empezó a diseñar modelos de acreditación de programas, que debía empezar por que las universidades empiecen a ordenar sus formas de gestión basado en procesos, y con ello afrontar el proceso de acreditación de programas de estudio; proceso muy difícil de conseguir, si no ordenas los procesos de gestión y muchos de los programas acreditados en el Perú una vez acreditados no pudieron mantener lo declarado en el proceso de acreditación, porque la estructura universitaria eran islas y los programas hacían su mejor esfuerzo para cumplir los estándares requeridos.

En la actualidad el modelo de acreditación de programas de estudio gestionado por SINEACE, considera en el factor 3: Aseguramiento de la Calidad, Estándar 7 la implementación de un Sistema de Gestión de la Calidad, y en la condición I: Existencia de objetivos académicos, grados y títulos a otorgar y planes de estudios correspondientes, Indicador 7: Plan de gestión de la calidad o Plan de mejora continua, 
orientado a elevar la calidad de la formación académica. Ambos modelos consideran que es muy importante ordenar la universidad usando el enfoque basado en procesos y aplicando mejora continua en la gestión universitaria y la mayoría de universidades no implementan un sistema de gestión debido a que, las instituciones educativas universitarias están liderados con falta de una cultura de mejora continua y planificación de objetivos institucionales a largo plazo, en ellos debemos considerar en las universidades:

a) Las herramientas de gestión educativa no están alineados a los objetivos académicos, planes de estudio, proceso de selección a estudiantes, procedimientos administrativos, grados y títulos de los programas académicos. Se evidencia en la falta de promoción y apoyo por la alta dirección.

b) La oferta académica cuyo servicio educativo requiere recursos para financiar sus actividades académicas y administrativas, debido a que el Plan Estratégico Institucional no guarda relación con los Planes Operativos Institucionales, y es porque en la mayoría de los casos para su construcción no participaron los dueños de los procesos sino es una copia de años anteriores reflejando presupuesto y financiado inconsistente.

c) La antropometría define el tipo de ambientes, mobiliario y equipamiento, al realizar el análisis de aulas, bibliotecas, talleres, laboratorios entre otros se encuentra que la infraestructura y equipamiento no está adecuado al cumplimiento de sus funciones a académicas ya que no cumplen con requisitos mínimos de seguridad, capacidad y equipamiento necesario. Los estudiantes de educación superior universitaria deben contar con locales de uso exclusivo que garantice el cumplimiento de los objetivos académicos.

La universidad es una comunidad académica orientada a la investigación y a la docencia, que brinda una formación humanista, científica y tecnológica, para ello se debe identificar e implementar con recursos necesarios, las líneas de investigación deben estar implementados para promover la investigación bajo la dirección de sus docentes, 
estudiantes en un ambiente propio para la creación, difusión de conocimiento ya que la razón de ser la universidad (Gordillo et al. 2020). Existen organismos como CONCYTEC que financian, asesoran en lo relacionado a Investigación, Innovación y Desarrollo.

Las universidades deben contar con personal docente calificado y como mínimo el 25\% debe contar al menos con un contrato a tiempo completo, para ello se evaluará el CAP (Cuadro de Asignación de Personal) versus las necesidades de las Escuelas Profesionales, y en base a ello asegurar la mejora del aseguramiento de la calidad (Murillo-Vargas et al. 2021).

La formación de estudiantes es integral y en las universidades se debe contar con servicios básicos complementarios como: servicio médico, social, psicopedagógico, deportivo entre otros los cuales facilite el aspecto formativo para ello la universidad deberá proveer recursos y así implementar y mantener los servicios, debe asegurarse que todos los estudiantes, de todos los programas y en todos sus locales reciban el mismo servicio (Mapén-Franco et al. 2020).

En razón de lo expuesto, la investigación tiene por objetivo determinar el impacto que ejerce la implementación de un Sistema de Gestión de la Calidad ISO 9001:2015 en el proceso de licenciamiento institucional de la Universidad Nacional Daniel Alcides Carrión.

\section{MÉTODO}

La investigación es de tipo explicativa porque hay una relación causal entre las variables, para mejorar la gestión de la Universidad Nacional Daniel Alcides Carrión, es necesario proponer la Implementación de un Sistema de Gestión de la Calidad ISO 9001:2015. Se conformó aleatoriamente un grupo $(\mathrm{R})$, constituido por un proceso en la Universidad, al prototipo (G1) al que se le aplica un estímulo o tratamiento experimental, el sistema de gestión de la calidad $(X)$ se aplica una prueba posterior al tratamiento $(01)$.

A un segundo grupo (G2), también conformado aleatoriamente, al que no se le suministra tal estimulo, el mismo que sirve únicamente como grupo de control, en forma simultánea se aplica una prueba (02 ). En ambos casos se asegura la representatividad estadística 
de los grupos.

\section{Estrategias de prueba de hipótesis}

Para la prueba de hipótesis se realiza mediante los siguientes pasos:

I. Se plantea la hipótesis nula y la alternativa.

II. Se selecciona el nivel

III. Se identifica el estadístico de prueba.

IV. Se forma la regla de decisión.

V. Se toma una muestra y se decide.

VI. No se rechaza Ho

VII. Se rechaza $\mathrm{Ho}$ y se acepta $\mathrm{Hi}$

El propósito de la prueba de hipótesis no es cuestionar el valor calculado del estadístico (muestral) sino hacer un juicio con respecto a la diferencia entre estadístico de muestra y un valor planteado del parámetro.

\section{Análisis de la información}

En el trabajo de investigación se usó el simulador Bizagi, además observaciones de procesos críticos de la gestión de la calidad en la Universidad. La metodología de BPM simulado en el software Bizagi, como instrumento de recojo de información, con el cual se mide la confiabilidad de información.

Para el procesamiento de datos hacemos uso de la estadística, y los datos obtenidos del simulador de Bizagi representados en procesos del sistema de gestión de calidad corrido en dos grupos, control y experimental.

\section{Procedimiento de la investigación}

Se analizó el rendimiento de los incidentes de la gestión de riesgos del proceso de gestión docente que es uno de los procesos clave del sistema de gestión de calidad de la Universidad Nacional Daniel Alcides Carrión, identificamos un grupo de control y un grupo 
experimental, para el grupo de control los indicadores de rendimiento se aplican en la situación actual, para luego evaluar los resultados del grupo experimental diseñamos un Modelo de gestión de la calidad ISO 9001:2015. Mediante la herramienta llamada Bizagi se modelo y se simuló el proceso de gestión de riesgos para el grupo de control y grupo experimental, construimos los procesos usando BPM.

El cálculo del tiempo de espera se realizó simulando los procesos en el software Bizagi de cada una de las actividades que conforman tal proceso. Se calculó el tiempo promedio de duración de la gestión del riesgo y con esa información optimizamos tiempos innecesarios e improductivos. Por lo tanto, se presenta:

En la figura 1, se indica el proceso de distribución académica, el cual inicia cuando el Director de Escuela elabora la distribución académica validando a los docentes activos en el periodo académico actual, en el caso que necesite plazas docentes solicitara la asignación correspondiente a Pedagogía universitaria, quien proporciona los docentes, una vez elaborado la distribución de plazas solicita la aprobación y validación de Pedagogía con lo que se da inicio a las actividades académicas.

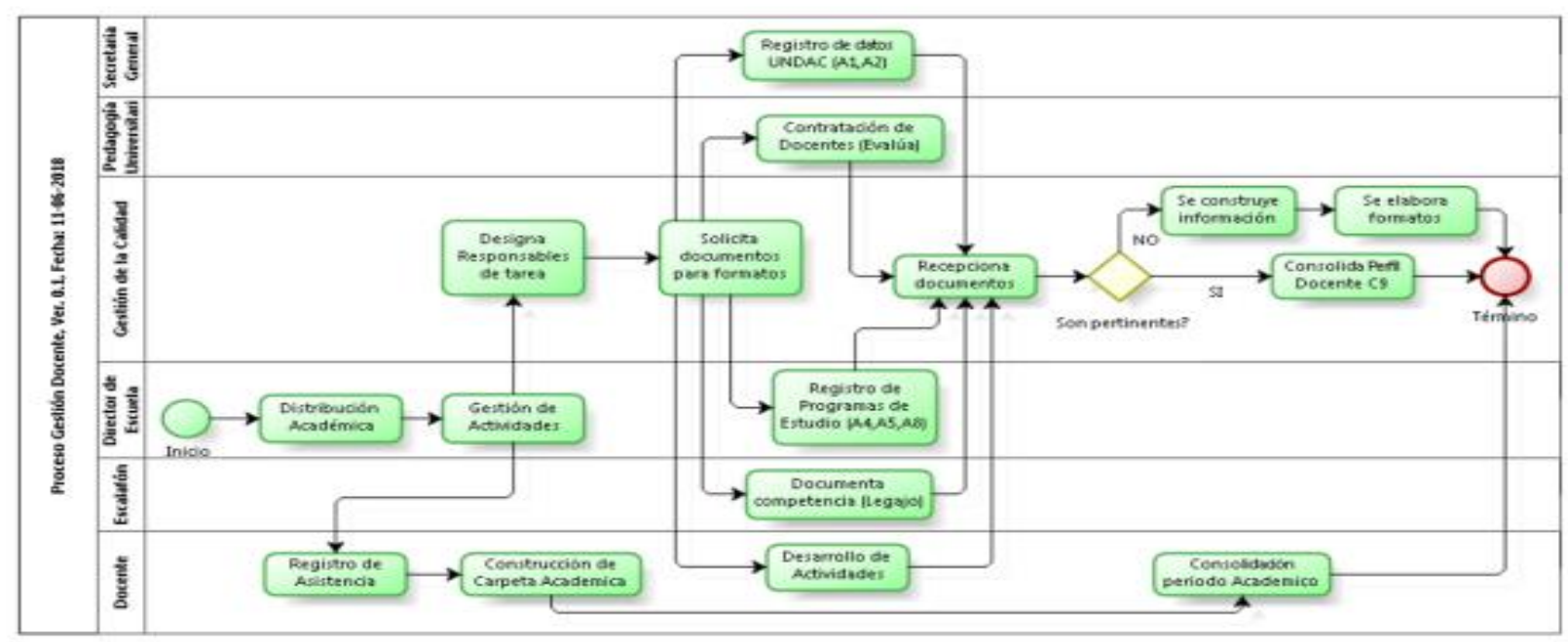

Figura 1. Proceso de gestión docente.

Fuente: El autor. 
En la figura 2, se evidencia el desarrollo de actividades, el cual inicia cuando el Director designa actividades a los docentes hábiles, el docente desarrolla la actividad encomendada y cuando termina presenta los entregables, el Director recepciona y valida la actividad.

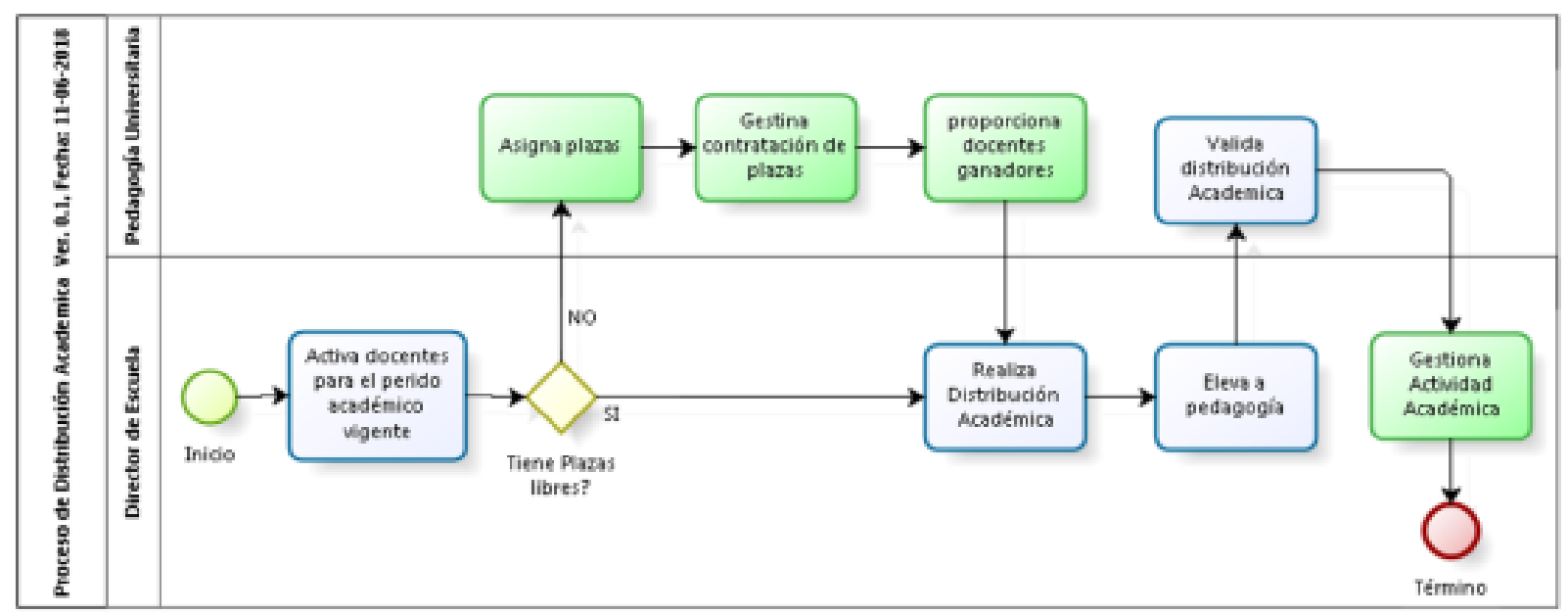

Figura 2. Proceso de distribución académica. Fuente: El autor.

En la figura 3, se evidencia la designación de responsabilidades, el cual inicia cuando Gestión de Calidad realiza un análisis de requerimiento, designa responsables de tarea, secretaria general emite resolución de designación, Gestión de Calidad solicita los documentos para el llenado de formatos, las oficinas remiten la documentación y en caso de no contar con ello realiza el informe correspondiente, para luego la oficina de gestión de la calidad realice el consolidado. 


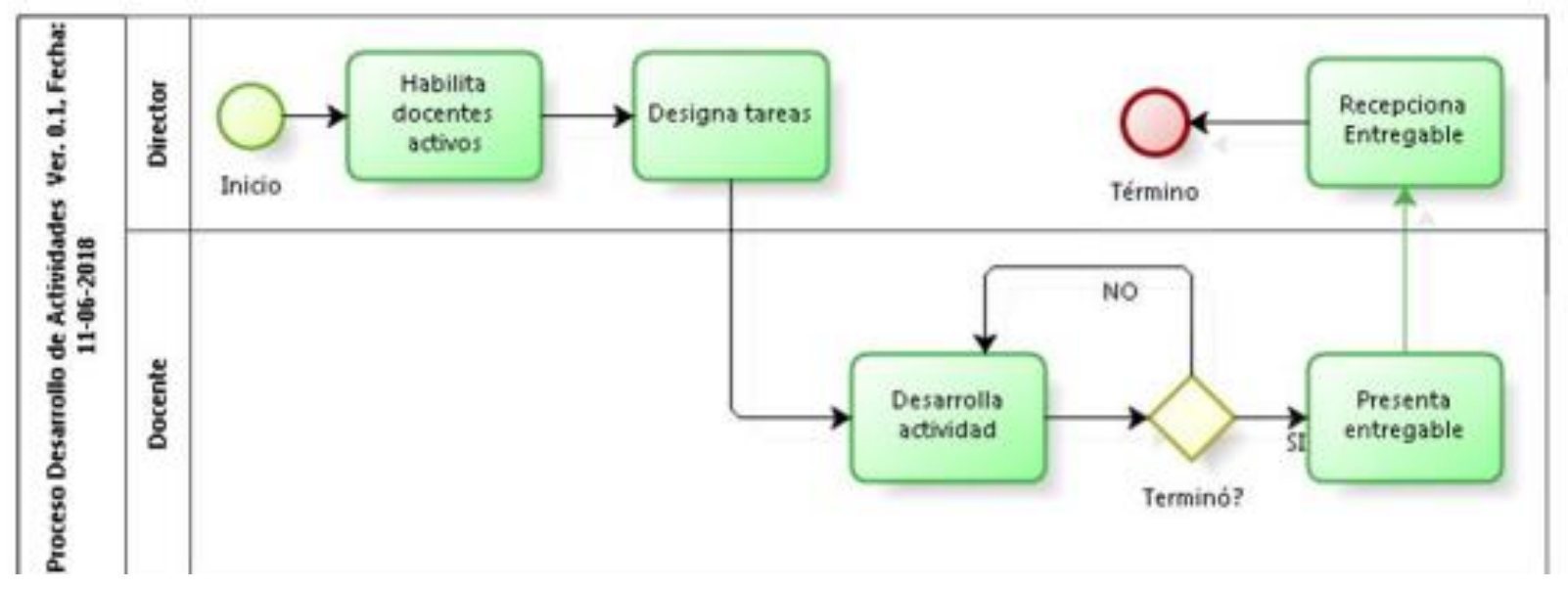

Figura 3. Proceso de desarrollo de actividades.

Fuente: El autor.

En la figura 4, se muestra el registro de datos UNDAC, A1, A2, Inicia cuando Gestión de Calidad codifica la sede, Filiales y Locales, luego solicita la documentación pertinente para llenado de formatos, secretaria general remite la documentación, en caso de no contar con ellos solicita los documentos a la Oficina de Obras, quien remite la documentación y en caso de no contar con ello solicita a la Dirección de Planificación para que emita la documentación en caso de no tener la documentación debe informar a la oficina de Gestión de Calidad para que realice el consolidado correspondiente, finalmente llena los formatos $A 1$ y A2. 
Revista Interdisciplinaria de Humanidades, Educación, Ciencia y Tecnología

Año VII. Vol. VII. N². Edición Especial II. 2021

Hecho el depósito de ley: pp201602FA4721

ISSN-L: 2542-3029; ISSN: 2610-802X

Universidad Nacional Experimental Francisco de Miranda (UNEFM). Santa Ana de Coro. Venezuela

Percy Ramírez-Medrano

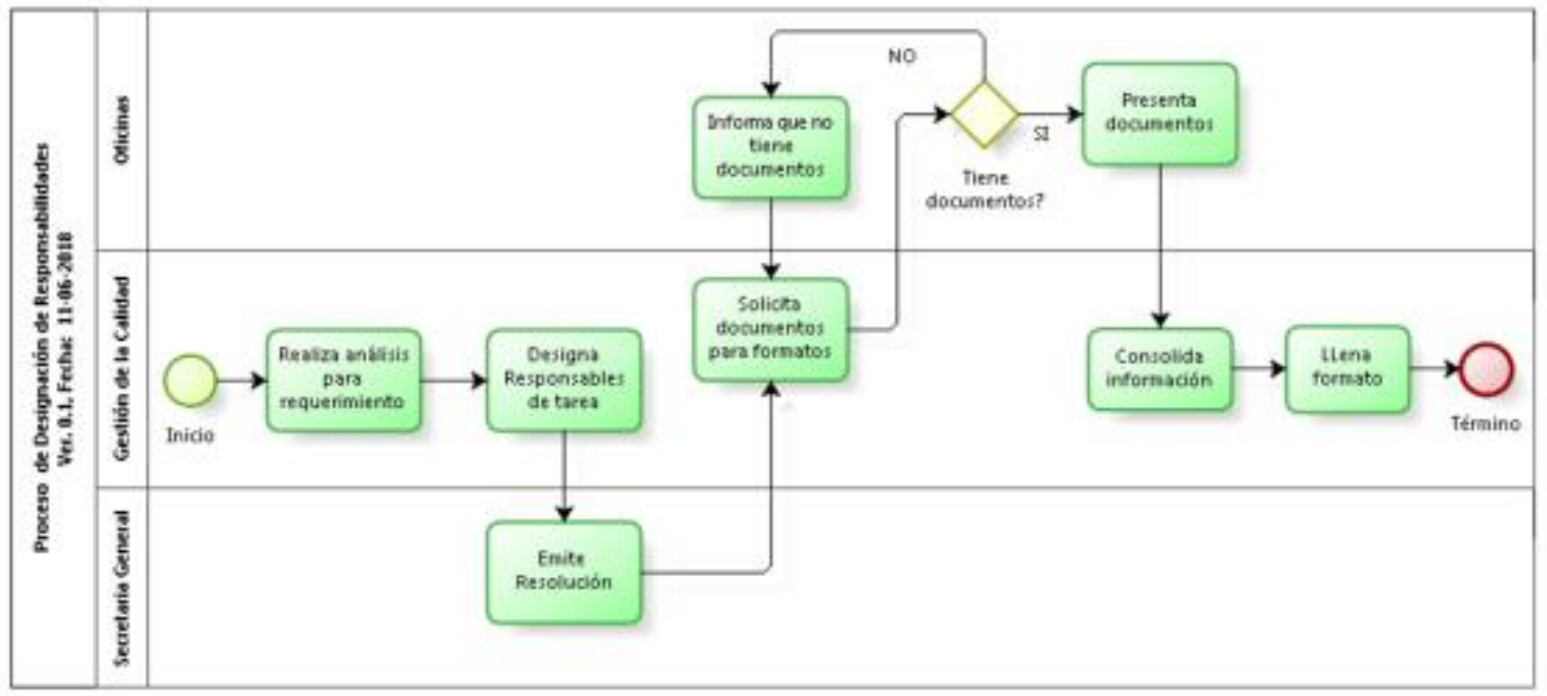

Figura 4. Proceso de designación de responsabilidades.

Fuente: El autor.

En la figura 5, se encuentra el registro de programas de estudio A4, A5, A8, Inicia cuando Gestión de la Calidad genera los códigos de los programas de estudio, especialidades por local, solicita documentos a Dirección de Escuela, Admisión, Posgrado e informática referente a programas de estudio, alumnos y en el caso que estas oficinas no tengan la documentación pertinente realizan un informe, con esta información Gestión de Calidad realiza la consolidación e imprime los formatos A4, A5, A8. 
Revista Interdisciplinaria de Humanidades, Educación, Ciencia y Tecnología

Año VII. Vol. VII. N². Edición Especial II. 2021

Hecho el depósito de ley: pp201602FA4721

ISSN-L: 2542-3029; ISSN: 2610-802X

Universidad Nacional Experimental Francisco de Miranda (UNEFM). Santa Ana de Coro. Venezuela

Percy Ramírez-Medrano

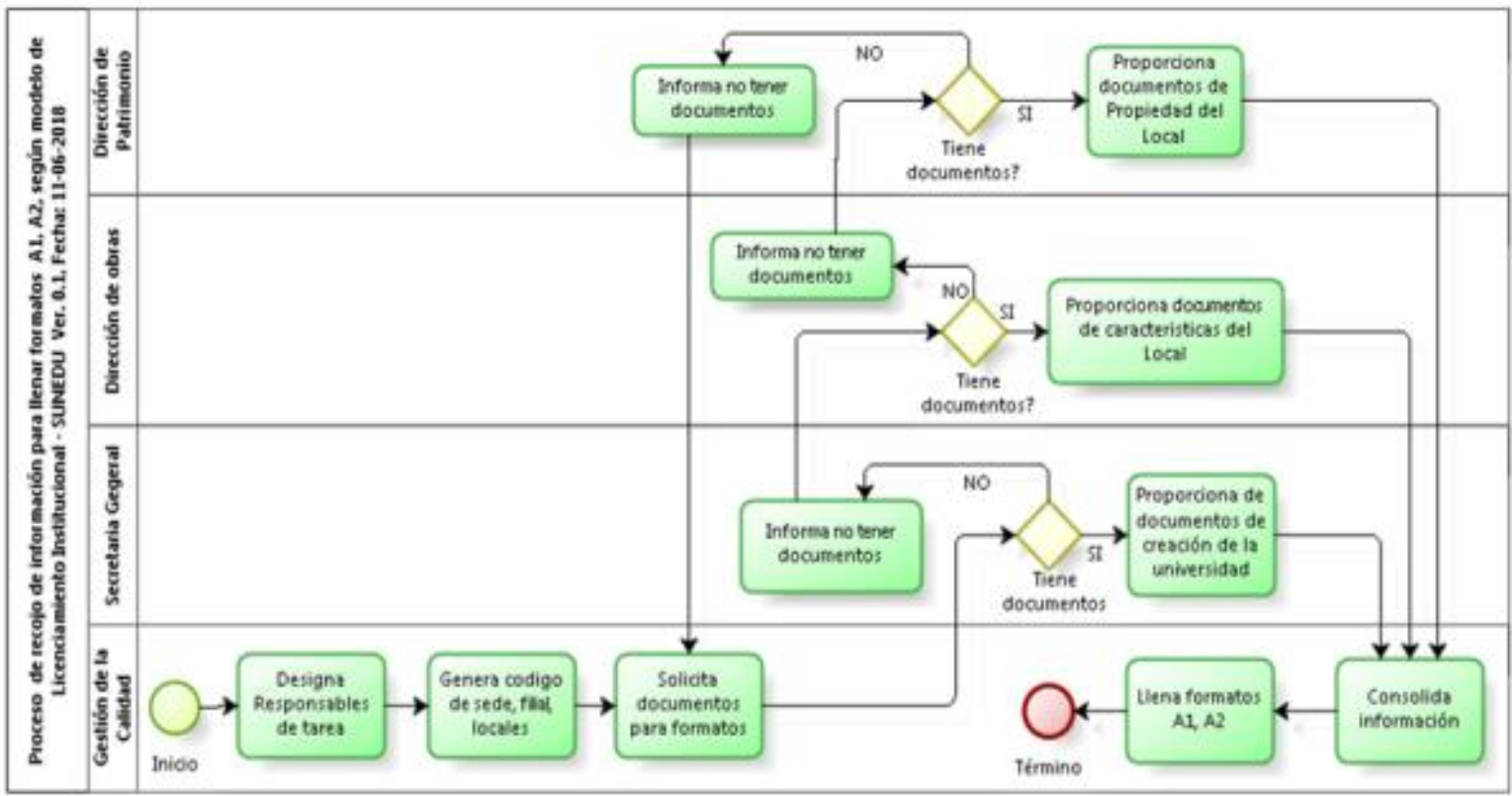

Figura 5. Proceso de recojo de información para llenar formato A1, A2. Fuente: El autor.

En la figura 6, se documenta competencia (legajo), Inicia cuando Secretaria General emite resolución de vínculo laboral docente, el docente presenta su C.V. documentado a la oficina de Escalafón. El Director de Escuela para la elaboración de distribución académica y otras actividades solicita el C.V. descriptivo a Escalafón, el docente debe actualizar sus documentes según se capacite. 
Revista Interdisciplinaria de Humanidades, Educación, Ciencia y Tecnología

Año VII. Vol. VII. N². Edición Especial II. 2021

Hecho el depósito de ley: pp201602FA4721

ISSN-L: 2542-3029; ISSN: 2610-802X

Universidad Nacional Experimental Francisco de Miranda (UNEFM). Santa Ana de Coro. Venezuela

Percy Ramírez-Medrano

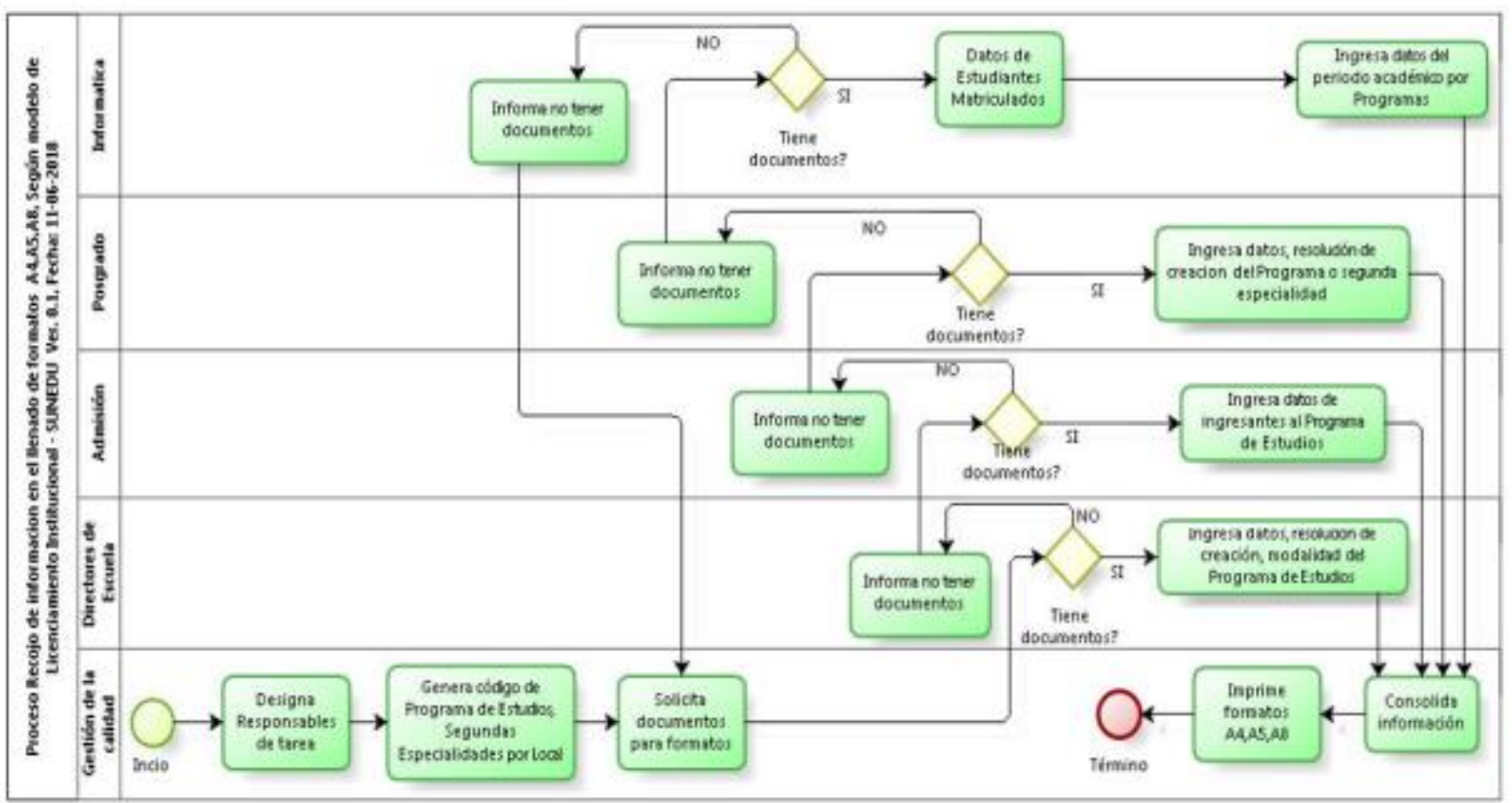

Figura 6. Proceso de recojo de información para llenar formato A4, A5, A8. Fuente: El autor.

En la figura 7, Consolida perfil docente C9, Inicia cuando Gestión de Calidad solicita documentos para el llenado de C9 a Director de Escuela, Escalafón quienes proporcionan información, en el caso de no contar con los documentos coordinan con los docentes y Gestión de Calidad llena el formato C9. 
Revista Interdisciplinaria de Humanidades, Educación, Ciencia y Tecnología

Año VII. Vol. VII. N². Edición Especial II. 2021

Hecho el depósito de ley: pp201602FA4721

ISSN-L: 2542-3029; ISSN: 2610-802X

Universidad Nacional Experimental Francisco de Miranda (UNEFM). Santa Ana de Coro. Venezuela

Percy Ramírez-Medrano

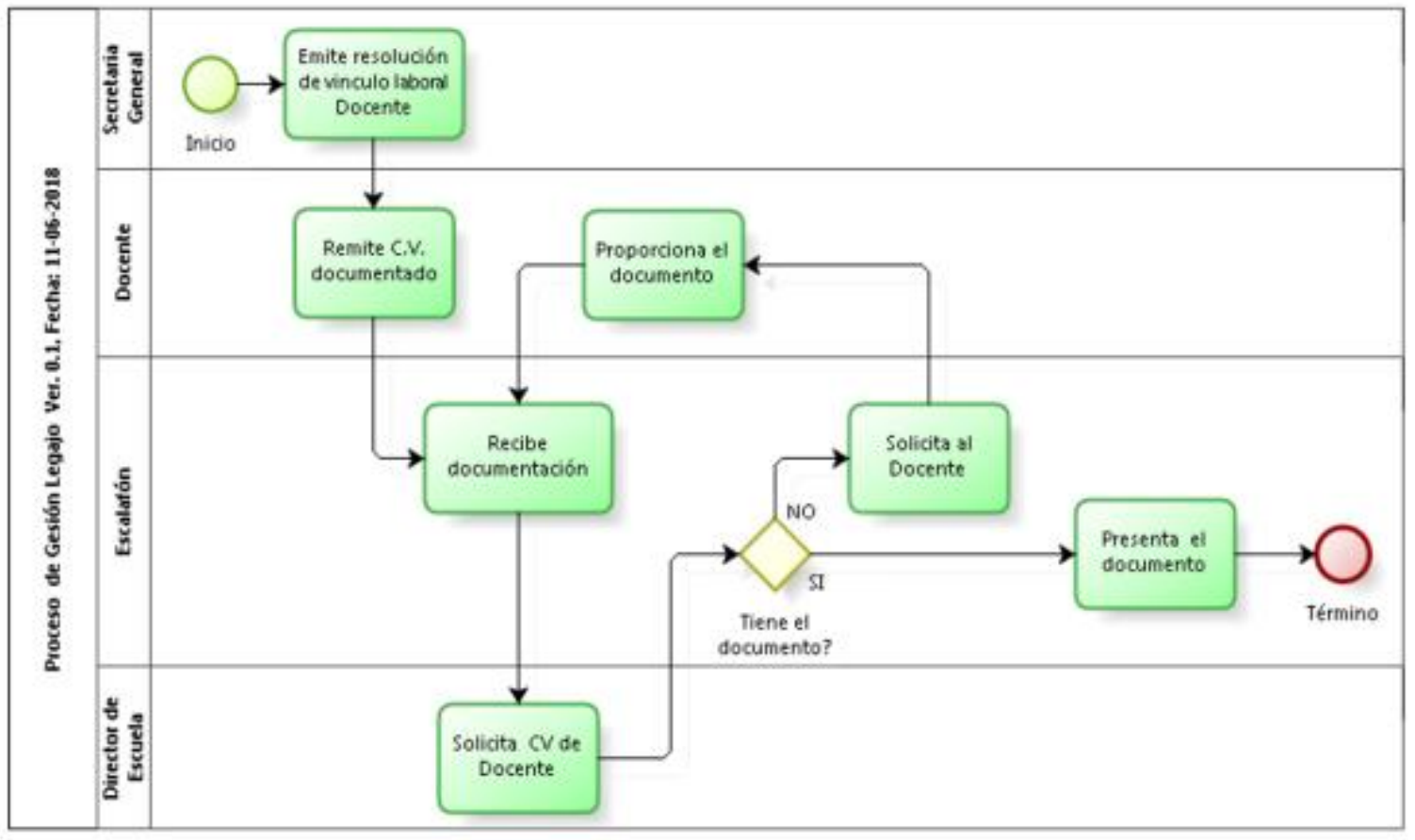

Figura 7. Proceso de gestión legajo.

Fuente: El autor.

Otros procesos desarrollados, se corresponden al:

a) Registro de asistencia, inicia cuando el Vicerrector Académico apertura el semestre académico, Pedagogía Universitaria genera directivas, Director de Escuela elabora los horarios de actividades académicas - administrativas, los docentes registran su asistencia, los cuales son revisados por los Directores de Escuela, Recursos Humanos realiza la consolidación para el pago correspondiente.

b) Desarrollo de actividades, inicia cuando el Director de Escuela designa tareas, los docentes detallan las actividades académicas, y administrativas y remiten los entregables correspondientes al Director de Escuela quien valida y genera un reporte.

c) Consolidación Académica, inicia cuando Pedagogía Universitario genera 
directivas, los Directores monitorean el desarrollo de actividades académicas según la construcción de la carpeta académica por los docentes, realizando un informe de evaluación y conformidad de la actividad por programa de estudios, con los informes Pedagogía Universitaria realiza el consolidado final.

\section{RESULTADOS}

Se presentan los resultados de la aplicación del tratamiento de la investigación:

\section{Cuadro 1.}

Prueba t Student para medias independientes.

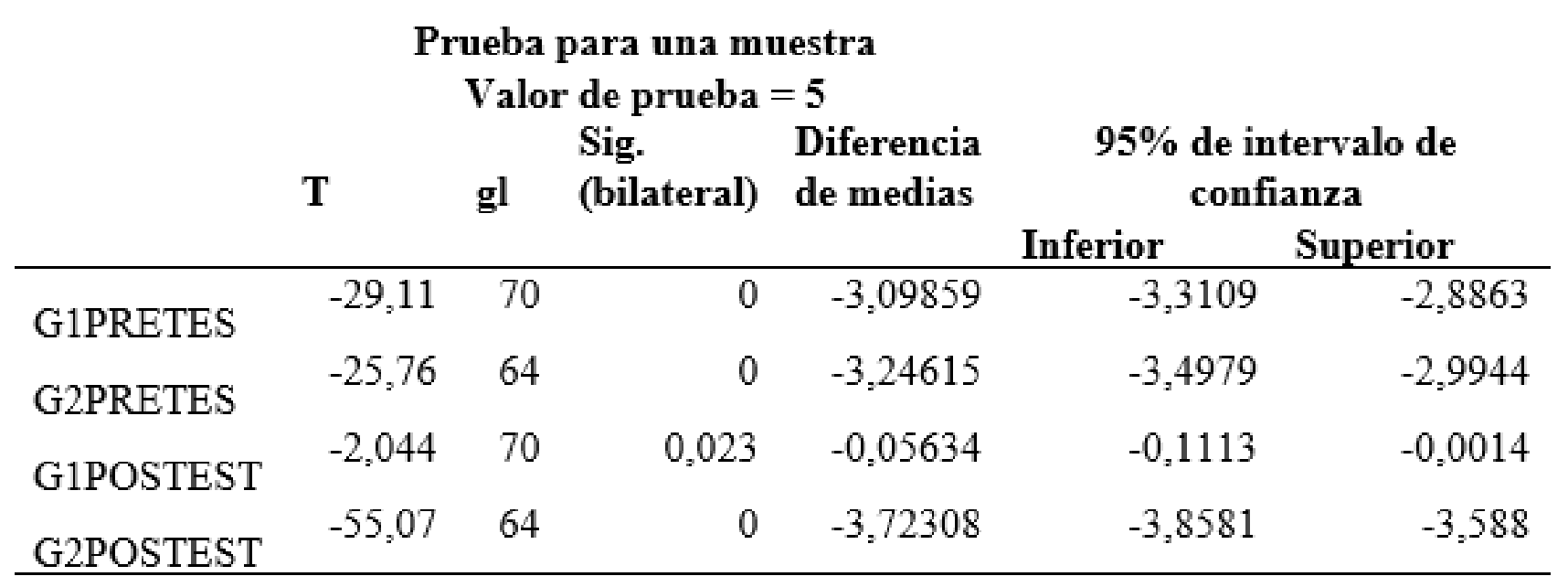

Fuente: El autor.

Al existir cambio estadístico en $\mathrm{G} 1$ en momento de postest, en comparación con G2 en el mismo momento, el cual permanece en posición neutral, se destaca que el tratamiento aplicado ejerció modificación sobre la gestión de calidad, llevada a cabo por la población estudiad, por lo tanto, se acepta $\mathrm{H} 1$ y se rechaza $\mathrm{H} 0$. 


\section{Prueba de hipótesis}

H1: La implementación de un Sistema de Gestión de la Calidad ISO 9001:2015 es efectivo en el proceso de licenciamiento institucional de la Universidad Nacional Daniel Alcides Carrión.

H0: La implementación de un Sistema de Gestión de la Calidad ISO 9001:2015 no es efectivo en el proceso de licenciamiento institucional de la Universidad Nacional Daniel Alcides Carrión.

\section{DISCUSIÓN}

El resultado concuerda con lo planteado por (León-Ramentol et al. 2018), quienes indican que el sistema de gestión contribuye al logro de la calidad de la universidad en conformidad a los criterios prescritos por las instituciones autorizadas para la acreditación institucional y del buen funcionamiento de la educación. Así mismo, (Huapaya-Capcha, 2019), admite que, en el Perú, en los últimos años, se ha iniciado un proceso de aseguramiento de la calidad de la educación por medio de implementar la mejora continua como un sistema cooperador para realizar ajustes durante el recorrido gerencial. Este estilo de sistema contribuye a fortalecer la acción docente, investigación y vinculación social, contribuyendo a centrarse en estándares internacionales como punto de encuentro para generar la satisfacción del cliente (Fontalvo \& De-La-Hoz, 2018); una de las ventajas para lograr tal fin, es que se generan lideres para poder atender las necesidades internas de la universidad, siendo considerable tener en cuenta, las empresas acreditas a través de la norma ISO, se constituyen en más competitivas en el mercado (Benzaquen-de-las-Casas, 2018), al formalizar sus procesos internos (SaltosCruz et al. 2017). 


\section{CONCLUSIÓN}

La implementación del Sistema de Gestión de la Calidad ISO 9001:2015 contribuye a que la Universidad Nacional Daniel Alcides Carrión pueda gestionar sus actividades académicas y administrativas con un enfoque basado en procesos con información relevante y medible, y a la vez permite retroalimentar los procesos de gestión de la calidad para determinar los procesos críticos y gestionar los riesgos y aplicar las propuestas de solución, para así lograr la mejora continua en la gestión de la calidad en beneficios de los estudiantes, docentes, personal administrativo, grupos de interés, partes interesadas y demás relacionados con el que hacer universitario además le permite un posicionamiento en la sociedad.

\section{REFERENCIAS CONSULTADAS}

Acreditación de programas de estudio gestionado por SINEACE. Recuperado desde https://n9.cl/qgiz

Benzaquen-de-las-Casas, J. (2018). La ISO 9001 y la administración de la calidad total en las empresas peruanas. Revista Universidad y Empresa, 20(35), 281312. https://doi.org/10.12804/revistas.urosario.edu.co/empresa/a.6056

Fontalvo, T., \& De-La-Hoz, E. (2018). Design of a Quality Management System ISO 9001:2015 in a Colombian University. Formación universitaria, 11(1), 3544. https://dx.doi.org/10.4067/S0718-50062018000100035

Gordillo, L., Domínguez, B., Vega, C, De la Cruz, A, \& Angeles, M. (2020). El marketing educativo como estrategia para la satisfacción de alumnos universitarios. Propósitos $\quad$ Representaciones, 8(spe), e499. https://dx.doi.org/10.20511/pyr2020.v8nspe1.499

Huapaya-Capcha, Y. (2019). Gestión por procesos hacia la calidad educativa en el Perú [Management by processes towards educational quality in Peru]. Revista Arbitrada Interdisciplinaria Koinonía, 4(8), 243-261. http://dx.doi.org/10.35381/r.k.v4i8.277 
León-Ramentol, C, Menéndez-Cabezas, A, Rodríguez-Socarrás, I, López-Estrada, B, García-González, M, \& Fernández-Torres, S. (2018). Importancia de un sistema de gestión de la calidad en la Universidad de Ciencias Médicas [Importance of a quality management system at the university of medical sciences]. Revista Archivo Médico de Camagüey, 22(6), 843-857.

Ley universitaria 30220. Recuperado desde https://n9.cl/gnr3

Mapén-Franco, F, Becerra Hernández, A, \& Martínez Prats, G. (2020). Importancia y satisfacción de los servicios universitarios en posgrado desde la perspectiva estudiantil. Revista San Gregorio,
26. https://doi.org/doi.org/10.36097/rsan.v1i38.1238

Murillo-Vargas, G, González-Campo, C., \& Piñeros, A. (2021). Modelo de evaluación del bienestar estudiantil universitario en Colombia. Formación universitaria, 14(2), 133-140. https://dx.doi.org/10.4067/S0718-50062021000200133

Saltos-Cruz, J. G., Jiménez Castro, W. F., \& López Miller, Z. E. (2017). Los Sistemas de Gestión de Calidad y la conformidad de la norma: un caso de estudio del sector de producción de plantas de calzado, República del Ecuador. Revista Publicando, 4(11 (2), 620-644.

(O2021 por los autores. Este artículo es de acceso abierto y distribuido según los términos y condiciones de la licencia Creative Commons Atribución-NoComercial-Compartirlgual 4.0 Internacional (CC BY-NC-SA 4.0) (https://creativecommons.org/licenses/by-nc-sa/4.0/ 